\title{
Laser-Ablative Synthesis of Ultrapure Magneto-Plasmonic Core-Satellite Nanocomposites for Biomedical Applications
}

\author{
Anton A. Popov 1,2,*, Zaneta Swiatkowska-Warkocka ${ }^{3}{ }^{(D}$, Marta Marszalek $^{3}\left(\mathbb{D}\right.$, Gleb Tselikov $^{2,4}{ }^{(D)}$, \\ Ivan V. Zelepukin ${ }^{1,5} \mathbb{D}^{\text {, }}$, Ahmed Al-Kattan ${ }^{2}\left(\mathbb{D}\right.$, Sergey M. Deyev ${ }^{1,5} \mathbb{D}$, Sergey M. Klimentov ${ }^{1}$, Tatiana E. Itina 6 \\ and Andrei V. Kabashin 1,2,*
}

1 Institute of Engineering Physics for Biomedicine (Phys-Bio), Moscow Engineering Physics Institute, 115409 Moscow, Russia; ivan.zelepukin@gmail.com (I.V.Z.); deyev@ibch.ru (S.M.D.); smklimentov@mephi.ru (S.M.K.)

2 Laboratory of Lasers Plasmas and Photonic Processing, CNRS, Aix-Marseille University (Campus of Luminy), 13288 Marseille, France; celikov@physics.msu.ru (G.T.); ahmed.al-kattan@univ-amu.fr (A.A.-K.)

3 Institute of Nuclear Physics, Polish Academy of Sciences, 31342 Kraków, Poland; zaneta.swiatkowska@ifj.edu.pl (Z.S.-W.); marta.marszalek@ifj.edu.pl (M.M.)

4 Center for Photonics and 2D Materials, Moscow Institute of Physics and Technology, 141700 Dolgoprudny, Russia

5 Shemyakin-Ovchinnikov Institute of Bioorganic Chemistry, Russian Academy of Sciences, 117997 Moscow, Russia

check for updates

Citation: Popov, A.A.; Swiatkowska-Warkocka, Z.; Marszalek, M.; Tselikov, G.; Zelepukin, I.V.; Al-Kattan, A.; Deyev, S.M.; Klimentov, S.M.; Itina, T.E.; Kabashin, A.V. Laser-Ablative Synthesis of Ultrapure Magneto-Plasmonic Core-Satellite Nanocomposites for Biomedical Applications. Nanomaterials 2022, 12, 649. https://doi.org/10.3390/nano 12040649

Academic Editor:

Vincenzo Amendola

Received: 1 January 2022

Accepted: 11 February 2022

Published: 15 February 2022

Publisher's Note: MDPI stays neutral with regard to jurisdictional claims in published maps and institutional affiliations.

Copyright: (C) 2022 by the authors. Licensee MDPI, Basel, Switzerland. This article is an open access article distributed under the terms and conditions of the Creative Commons Attribution (CC BY) license (https:// creativecommons.org/licenses/by/ $4.0 /)$.
6 Hubert Curien Laboratory (UMR CNRS 5516), Jean Monnet University, 42000 Saint-Etienne, France; tatiana.itina@univ-st-etienne.fr

* Correspondence: aapopov1@mephi.ru (A.A.P.); andrei.kabashin@yahoo.com (A.V.K.)

\begin{abstract}
The combination of magnetic and plasmonic properties at the nanoscale promises the development of novel synergetic image-guided therapy strategies for the treatment of cancer and other diseases, but the fabrication of non-contaminated magneto-plasmonic nanocomposites suitable for biological applications is difficult within traditional chemical methods. Here, we describe a methodology based on laser ablation from Fe target in the presence of preliminarily ablated water-dispersed $\mathrm{Au}$ nanoparticles (NPs) to synthesize ultrapure bare (ligand-free) core-satellite nanostructures, consisting of large (several tens of $\mathrm{nm}$ ) Fe-based core decorated by small (mean size $7.5 \mathrm{~nm}$ ) Au NPs. The presence of the Fe-based core conditions a relatively strong magnetic response of the nanostructures (magnetization of $>12.6 \mathrm{emu} / \mathrm{g}$ ), while the Au NPs-based satellite shell provides a broad extinction peak centered at $550 \mathrm{~nm}$ with a long tale in the near-infrared to overlap with the region of relative tissue transparency $(650-950 \mathrm{~nm})$. We also discuss possible mechanisms responsible for the formation of the magnetic-plasmonic nanocomposites. We finally demonstrate a protocol to enhance colloidal stability of the core-satellites in biological environment by their coating with different polymers. Exempt of toxic impurities and combining strong magnetic and plasmonic responses, the formed core-satellite nanocomposites can be used in biomedical applications, including photo- and magneto-induced therapies, magnetic resonance imaging or photoacoustic imaging.
\end{abstract}

Keywords: nanoparticles; laser ablation in liquids; gold; iron; core-satellite; IR hyperthermia; modeling

\section{Introduction}

Recent advances in the development of nanotechnologies have led to the emergence of novel diagnostic and therapeutic modalities based on new nanomaterials [1-3]. Much attention in this field is given to plasmonic nanoparticles (NPs), which are capable of providing a variety of phenomena, including resonant optical extinction (absorption + scattering) in the visible and near infrared (near-IR) range [4], strong local field enhancement [5], resistance to photobleaching, and highly efficient photothermal conversion [6]. Gold (Au) NPs presents the most explored plasmonic nanomaterial [7] due to its chemical stability, biocompatibility [8], and the availability of simple surface functionalization protocols via 
thiol chemistry [9]. Au NPs were successfully used in therapeutic modalities, including photo-induced hyperthermia [10] and in diagnostic modalities, including optical coherence tomography [11], photoacoustic tomography [12], and confocal reflectance microscopy [13]. Magnetic NPs represent another appealing class of nanomaterials for biomedical applications [14]. These NPs can be guided by external magnetic fields both in vitro and in vivo to enable a series of therapeutic and diagnostic modalities, including magnetic resonance imaging [15], magnetic separation of cells and proteins [16], controllable drug delivery [17], and magnetic hyperthermia [18]. Among magnetic materials, iron (Fe)-based NPs are considered to be the most promising due to their biocompatibility, biodegradability, and strong magnetic response [14].

The combination of plasmonic and magnetic functionalities within a single nanoformulation looks especially interesting as it promises a synergetic enhancement of involved therapeutic and diagnostic modalities. Fe-Au magneto-plasmonic nanostructures are typically composed of a Fe-based core, covered by an Au shell (Fe@Au core-shells), which makes possible both magnetic response and the generation of plasmonic extinction peak in the visible and near-IR range $[19,20]$. Core-satellite structures present one of the promising variations of Fe@Au core-shell geometry, in which relatively large $(>50 \mathrm{~nm})$ Fe-based NPs are decorated by a layer (layers) of small $(<10 \mathrm{~nm})$ Au NPs [21-26] (Fe@Au core-satellites). These structures not only enable magneto-plasmonic response, but also offer a large surface for the conjugation with various functional biomolecules, as the surface area of satellite material $(\mathrm{Au})$ is much larger compared to alternative structures of similar size (spherical nanoparticles, classical core-shells with completely covered core surface). However, advantages provided by the core-satellite structures are usually compromised by a high cost and complexity of synthesis procedures [21-23]. In fact, conventional chemical routes imply stepwise processes, in which preliminarily prepared $\mathrm{Au}$ and Fe oxide NPs are first functionalized by appropriate ligands, which renders possible a subsequent assembling of the core-satellites [24-26]. Such techniques provide relatively good control of size, composition, and structure of the Fe@Au core-satellites, but the use of ligands blocks the surfaces of Fe or $\mathrm{Au}$ (or both), which complicates their further conjugation with functional biomolecules and protective biopolymers. Moreover, biomedical applications of chemically synthesized NPs often have limitations due to the presence of toxic impurities originated from precursors or incomplete reactions. Another synthesis pathway is based on X-ray radiolysis of $\mathrm{HAuCl}_{4}$ in colloidal solutions of Fe oxide NPs in the presence of a number of different chemical substances [27], but this approach is also not free of residual contamination problems and requires sophisticated and costly equipment.

Pulsed laser ablation is an alternative nanofabrication pathway capable of producing nanostructures with various geometries, including the core-shell one. This approach is based on a natural production of nanoclusters during the action of laser radiation on a solid target [28]. When produced in a gaseous medium, the nanoclusters can be deposited on a substrate to form a thin nanostructured film [29], while pulsed laser ablation in liquids (PLAL) leads to the release of nanoclusters into the liquid medium to form a colloidal solution [30]. A high efficiency and cleanness of this method made it highly suitable for a plethora of applications in biomedicine [3,31,32]. Ultrashort laser ablation (femtosecond (fs) or picoseconds (ps)) has demonstrated the most efficient control of NPs among PLAL methods (see examples in [33-35]). Using these approaches, we recently synthesized a variety of nanomaterials, including $\mathrm{Au}[8,33], \mathrm{Si}[36], \mathrm{TiN}$ [37,38], organic materials [39], and demonstrated their great potential for biomedical tasks.

PLAL has already been explored in the synthesis of Fe-Au NPs with diverse geometries. Barcikowski et al. ablated Au-Fe alloy target and found that structure of the produced core-shell NPs depends on NPs size and nature of the liquid media [40,41], while Amendola et al. demonstrated that nanosecond laser ablation from an $\mathrm{Au}-\mathrm{Fe}$ alloy target or layers of alternating $\mathrm{Au}$ and Fe thin films in water and ethanol results in the formation of Fe-Au NPs of alloy or core-shell geometry [42,43]. It was shown that such NPs have 
remarkable magnetic and plasmonic properties and are promising for magnetic resonance imaging (MRI), SERS, and computer tomography (CT) applications [42].

Herein, we further extend the capabilities of laser-ablative methodology toward the synthesis of core-satellite magneto-plasmonic structures. Our approach is based on fs laser ablation from Fe target in aqueous colloidal solutions of small $(<10 \mathrm{~nm}) \mathrm{Au}$ NPs, preliminarily prepared by PLAL. We show that the formed $\mathrm{Fe}_{x} \mathrm{O}_{y} @ \mathrm{Au}(x, y$-variables $)$ core-satellites exhibit good magnetic properties and significant optical extinction in the window of biological tissue transparency. We then use different polymers to coat the core-shell nanocomposites and show that such a coating leads to their colloidal stabilization even under storage in high ionic strength solutions. Such nanocomposites are promising for multimodal magneto-optical biomedical applications.

\section{Materials and Methods}

\subsection{Synthesis of Nanoparticles}

For the synthesis of $\mathrm{Fe}_{x} \mathrm{O}_{y} @ \mathrm{Au}$ core-satellite nanocomposites we modified a two-step method of fs PLAL, which was previously developed by our group for the preparation of Gold-Silicon core-shell NPs [44,45]. A schematic diagram of the synthesis procedure is shown in Figure 1. During the first step we synthesized Au NPs with mean size $7.5 \mathrm{~nm}$ using radiation from the $\mathrm{Yb}: \mathrm{KGW}$ laser $(420 \mathrm{fs}, 1025 \mathrm{~nm}, 10 \mathrm{kHz}, 50 \mu \mathrm{J})$ focused by a $75 \mathrm{~mm}$ lens on surface of a gold target (99.99\%, GoodFellow, Delson, QC, Canada) immersed in $10 \mathrm{~mL}$ of $1 \mathrm{mM}$ aqueous $\mathrm{NaCl}$ solution, and placed $10 \mathrm{~mm}$ below the liquid surface. As previously demonstrated [46,47], the addition of $\mathrm{NaCl}$ to water improves colloidal stability of laser-synthesized Au NPs and provides in situ control of the mean size of Au NPs by adjustment of $\mathrm{NaCl}$ concentration. The Au target was continuously moved perpendicular to the laser beam at a speed of $2 \mathrm{~mm} / \mathrm{s}$ to avoid ablation from the same point. Duration of the synthesis was $20 \mathrm{~min}$. During the second step of the synthesis, a metallic Fe target (99.99\%, GoodFellow, Delson, QC, Canada) was placed at the bottom of the glass vessel filled with the colloidal solutions of Au NPs prepared in the first step and laser ablation was performed with the same laser, but with slightly modified parameters (repetition rate $5 \mathrm{kHz}$, pulse energy $100 \mu \mathrm{J}$ ) for 15 minutes. Ablated mass concentrations of $\mathrm{Au}$ and $\mathrm{Fe}$ were 0.1 and $0.05 \mathrm{~g} / \mathrm{L}$, respectively.

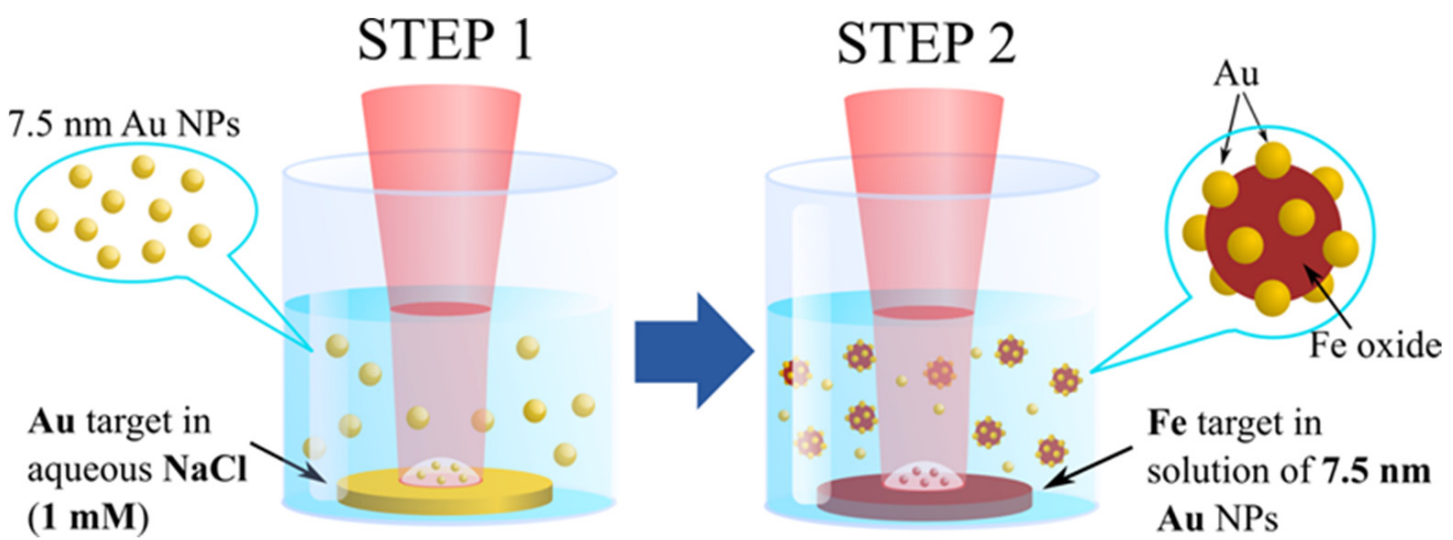

Figure 1. Schematic diagram of two-step PLAL synthesis of core-satellite Au-Fe NPs.

Non-reacted Au NPs were eliminated by magnetic separation step. For this a NdFeB magnet was placed for 5 min next to the bottom of the test tube filled with the colloidal solutions of NPs. The magnet attracted magnetic fraction of NPs to the bottom, while nonmagnetic NPs remained dispersed in the solution. Then the solution from the upper part of the test tube was discarded leaving about $20 \mu \mathrm{L}$ at the bottom and the test tube was refilled with fresh $1 \mathrm{mM}$ aqueous $\mathrm{NaCl}$ solution. The NPs remaining in the test tube were re-dispersed by an ultrasonication step. The procedure was repeated two times to ensure 
good separation of NPs. The obtained solutions were used for further experiments and characterization.

\subsection{Coating of Nanoparticles}

Polyethylenimine (PEI) branched $25 \mathrm{kDa}$, polyallylamine (PAA) hydrochloride $17.5 \mathrm{kDa}$, carboxymethyl-dextran (CMD) sodium salt, polyacrylic acid (PAAC) sodium salt $5.1 \mathrm{kDa}$, polyethylene glycol-silane (PEG-silane) $5 \mathrm{kDa}$, ammonium hydroxide $30 \%$ were purchased from Sigma-Aldrich (St. Louis, MO, USA).

NPs coating by CMD, PAAC, PAA, and PEI polymers was performed by coincubation procedure. Total of $200 \mu \mathrm{L}$ of NPs suspension was mixed with $200 \mu \mathrm{L}$ of $10 \% w / v$ solution of a polymer in distilled water. Then, the mixture was ultrasonicated, heated to $80^{\circ} \mathrm{C}$ for $1 \mathrm{~h}$, cooled to room temperature and the NPs were washed three times with water by centrifugation $(20,000 \mathrm{~g}, 20 \mathrm{~min})$.

NPs coating by PEG was performed by PEG-silane hydrolysis. For this, $1 \mathrm{mg}$ of NPs was centrifuged at 20,000 $\mathrm{g}$ for $20 \mathrm{~min}$ and redispersed in dry ethanol. After that $10 \mu \mathrm{L}$ of $30 \%$ hydroxide ammonia and $50 \mu \mathrm{L}$ of water were added to the suspension and NPs were thoroughly ultrasonicated. Then $100 \mu \mathrm{g}$ of PEG-silane in $100 \mu \mathrm{L}$ of dry ethanol was added dropwise, the tube was shaken and heated to $60^{\circ} \mathrm{C}$ for $2 \mathrm{~h}$. Then, NPs were washed by centrifugation $(20,000 \mathrm{~g}, 20 \mathrm{~min})$ forming unreacted chemicals 1 time with ethanol and 2 times with water.

\subsection{Characterization of Nanoparticles}

Morphology, structure, size, and composition of the synthesized NPs were characterized by a high resolution transmission electron microscope (HR-TEM, JEM 3010, JEOL, Tokyo, Japan) operating at $300 \mathrm{kV}$ and by a scanning electron microscope (SEM, MAIA 3, Tescan, Brno, Czech Republic) operating at $0.1-30 \mathrm{kV}$ coupled with an energy dispersive spectroscopy (EDS) detector (X-act, Oxford Instruments, Abingdon, UK). Samples for the electron microscopy were prepared by dropping $1 \mu \mathrm{L}$ of a NPs solution onto a carboncoated copper grid (for HR-TEM imaging) or onto a cleaned silicon substrate (for SEM imaging) and subsequent drying at ambient conditions.

$\zeta$-potential and hydrodynamic size were measured using a Zetasizer ZS instrument (Malvern Instruments, Malvern, UK) capable of measuring dynamic light scattering (DLS). Concentrations of NPs solutions were determined by measuring the ablation target weight before and after the ablation step and by dividing this mass difference by the ablation liquid volume.

Optical extinction spectra were measured by a UV-VIS spectrophotometer (UV2600, Shimadzu, Kyoto, Japan) using plastic cuvettes with $10 \mathrm{~mm}$ optical path length (for bare NPs) or in $100 \mu \mathrm{L}$ volume of 96-well plastic plate using Infinite M1000Pro (Tecan, Männedorf, Switzerland) microplate reader (for polymer coated NPs).

Magnetic properties of the NPs were measured using a superconducting quantum interference device (MPMS XL SQUID, Quantum Design, San Diego, CA, USA) magnetometer. Magnetic curves $\mathrm{M}(\mathrm{H})$, where $\mathrm{M}$ is the magnetization and $\mathrm{H}$ is the applied magnetic field, were measured at $\mathrm{T}=300$ and $5 \mathrm{~K}$. The zero-field cooling/field cooling (ZFC/FC) measurements at $\mathrm{H}=100$ Oe were also performed on the SQUID. In this purpose, the ZFC temperature dependences of the magnetization were measured by cooling the sample from 325 to $5 \mathrm{~K}$ and then applying a field of 100 Oe to record the magnetization, while the sample was heated from 5 to $325 \mathrm{~K}$. The field cooled (FC) measurements were made with a field of 100 Oe applied during the cooling process.

\subsection{Calculation of Optical Properties}

The extinction spectra of NPs were simulated using the electromagnetic Mie theory, in which the numerical solutions are presented as infinite series of several combinations of Bessel functions [48,49]. The numerical model was based on the implementation of Yang's algorithm [49] for multilayered spheres [50,51] with the possibility of calculating the optical 
response of an ensemble of independent particles with a normal size distribution. Thus, the analytical solution for the light scattering by a multilayered sphere was calculated by expressing the electromagnetic field inside each layer of the sphere as a linear combination of the inward and outward travelling waves. Each layer was characterized by a size parameter $x_{i}=2 \pi n_{m} r_{i} / \lambda=k r_{i}$ and by a relative refractive index $m_{i}=n_{i} / n_{m}, i=1,2, \ldots$, $L$. Here, $\lambda$ is the incident wavelength in vacuum, $r_{i}$ is the outer radius of the $i$ th layer, $k$ is the propagation constant, and $n_{m}$ and $n_{i}$ are the refractive index of the medium outside the particle and its $i$ th component, respectively, and $L$ is the total number of layers. $L=1$ for spherical $\mathrm{Au}$ and Fe oxide NPs, while $L=2$ for core-satellites.

\section{Results and Discussion}

\subsection{Synthesis and Structural, Optical and Magnetic Characterizations of Nanomaterials}

Core-satellites were synthesized by a two-step fs PLAL as described in Experimental Section and schematically shown in Figure 1. The prepared colloids of nanocomposites were almost black with a reddish-violet tint. To remove potentially unreacted Au NPs from the colloid we separated magnetically active NPs by applying external magnetic field. Before TEM and EDS imaging the colloidal solutions were additionally kept in a dialysis tube (Serva Visking ${ }^{\circledR} 44120$, pore diameter ca. $2.5 \mathrm{~nm}$, buffer volume $3 \mathrm{~L}$, Heidelberg, Germany) for $20 \mathrm{~h}$ to remove small amorphous iron oxide flakes.

The analysis of TEM images (Figure 2a) showed that most NPs had core-satellite architectures with higher contrast of satellites, which evidences the presence of Fe and $\mathrm{Au}$ in the core and satellites, respectively. At the same time, HR-TEM studies clearly demonstrated polycrystalline structure of both the core and the satellites (Figure $2 \mathrm{~b}$ ). Figure $2 c$ (inset) presents the chemical elemental mapping obtained by EDS technique in HR-TEM mode. The figure confirms that, the core was formed uniquely by iron-based compounds, while Au was presented only in satellites. As follows from size histogram depicted in Figure 2c, the size of Fe-based cores varied between 10 and several tens of $\mathrm{nm}$ with the mean size $30 \mathrm{~nm}$, while the mean size of Au satellites was $7.5 \mathrm{~nm}$. It should be noted that the core-shells nanostructures with Au core and Fe-based shell were also formed (data not shown), when large ( $>10 \mathrm{~nm}$ ) Au NPs were present in the colloid during the ablation of Fe target. Similar tendency was earlier observed and explained for the synthesis of Fe oxide@Au core@shell nanostructures by laser ablation of Au-Fe alloy targets in water [52].

Optical examination of the colloidal solutions (Figure 2d) revealed that the coresatellites exhibited a strong extinction peak associated with the excitation of plasmon resonance in $\mathrm{Au}$ NPs [4]. The position of this peak (green line in Figure 2d) was red shifted compared to the plasmonic feature of pure Au NPs centered at $521 \mathrm{~nm}$ (red line in Figure 2d). Indeed, the plasmonic peak of the core-satellites was centered at $550 \mathrm{~nm}$ and had a broad shoulder extending over $800 \mathrm{~nm}$, which largely overlaps with the optical window of relative tissue transparency $(650-950 \mathrm{~nm})$. This broadening of the plasmonic feature can have several origins, including the hybridization and coupling of plasmonic modes between Au NPs in their aggregates [53], the elongation of Au NPs [54], and the formation of the composite core-satellite nanostructures. The coupling between plasmonic modes of individual Au NPs could partly contribute to the spectrum broadening, although it cannot fully explain it, because for Au NPs the plasmonic coupling can redshift the position of the plasmon resonance up to $100 \mathrm{~nm}$ (to 620-630 nm) [55], while we observed broadening of the plasmonic feature up to $800 \mathrm{~nm}$. The elongation of Au NPs can be ruled out because TEM images (Figure S1) demonstrate that Au NPs are almost perfectly spherical, and their optical spectrum (Figure 2d, red line) has sharp plasmonic peak at 520 $\mathrm{nm}$ without any broadening. Therefore, we can conclude that the origin of the observed broadening of the plasmonic feature (Figure $2 \mathrm{~d}$, green line) must include the formation of core-satellites nanostructures, in which the core and the satellites are formed by dielectric Fe oxide and $\mathrm{Au}$, respectively. 
(a)
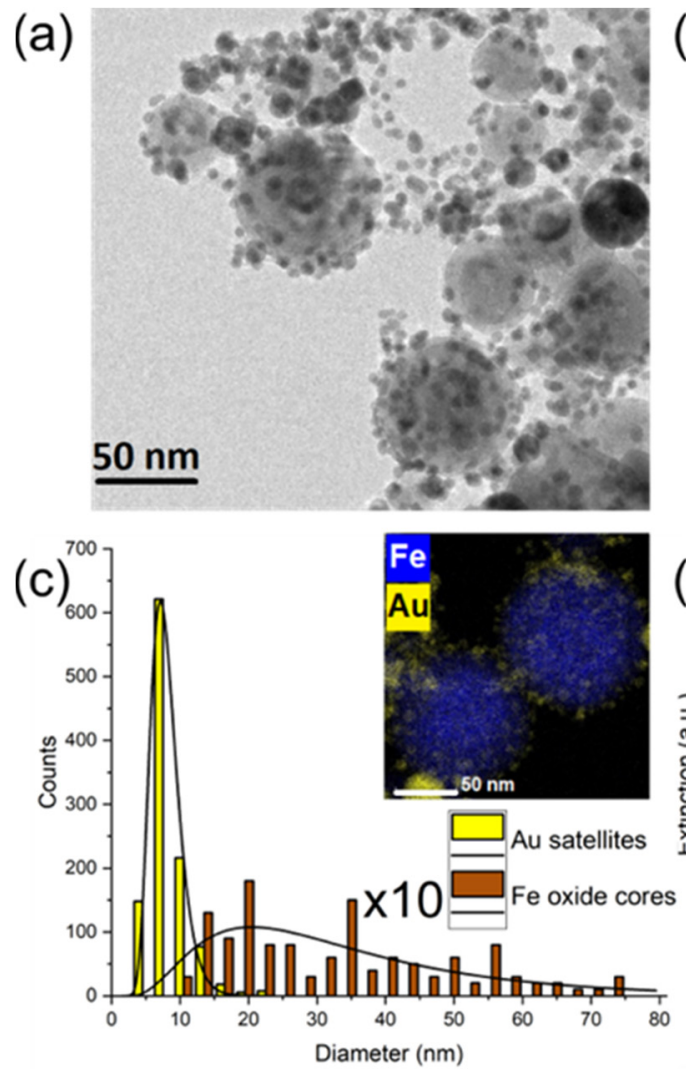

(b)
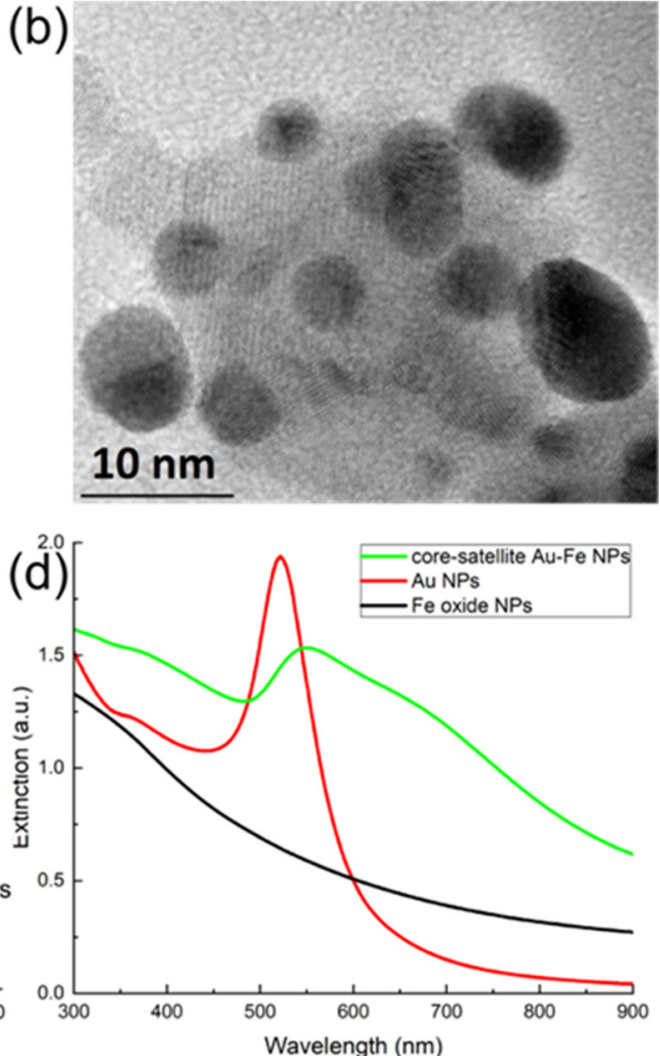

Figure 2. Characterization of core-satellite Au-Fe NPs. (a) TEM image of the NPs. (b) HR-TEM image of the NPs. (c) Size distribution of Au satellites (yellow) and Fe oxide cores (brown) (note that amount of cores is multiplied by 10). The inset demonstrates elemental mapping (Fe-blue, Au-yellow) of the NPs measured by EDX technique. (d) Optical extinction spectra of Fe oxide NPs (black curve), Au NPs (red curve) and core-satellite Au-Fe NPs (green curve).

We then carried out a series of calculations to simulate the observed plasmonic response. The numerical model was based on the implementation of Yang's algorithm for multilayered spheres. In the case of simple one-component NPs (pure Au or Fe oxide NPs) the solution corresponds to the classical Mie theory. In the case of core-satellites, we adopted the effective-medium Maxwell-Garnett approximation describing satellites as a layer (shell) with effective optical properties [56,57]. The effective shell consists of a matrix medium (water) with Au NPs as inclusions. The resulted spectra along with the calculation parameters are shown in Figure S2. The simulated extinction spectra are in a reasonable agreement with the experimental results. Good agreement was observed for the curves calculated for bare Fe oxide NPs and Au NPs. For the core-satellites, even though the applied approximation disregards coupling between resonant plasmonic modes of $\mathrm{Au}$ satellites, the general trend is still rather well reproduced, particularly the peak and the slow decay of the extinction coefficient with increase of wavelength.

We also investigated magnetic properties of the synthesized NPs. The results for pure laser-synthesized Fe oxide NPs and for the core-satellite nanostructures are summarized in Table 1. Hysteresis loops recorded at $5 \mathrm{~K}$ and $300 \mathrm{~K}$ are presented in Figure 3a. The hysteresis loops at both temperatures were open and clearly showed ferromagnetic behavior of the NPs. The coercive field at $5 \mathrm{~K}$ was slightly larger than at $300 \mathrm{~K}$. The saturation magnetization $\left(\mathrm{M}_{\mathrm{S}}\right)$ of core-satellites $\left(\mathrm{M}_{\mathrm{S}}=12.3 \mathrm{emu} / \mathrm{g}\right.$ at $\left.300 \mathrm{~K}\right)$ was significantly lower than $\mathrm{M}_{\mathrm{S}}$ of bulk magnetite $\left(92 \mathrm{emu} / \mathrm{g}\right.$ ) [58] or bulk maghemite (76 emu/g) [59]. The $\mathrm{M}_{\mathrm{S}}$ of pure laser-ablated Fe oxide NPs was $44.7 \mathrm{emu} / \mathrm{g}$ at $300 \mathrm{~K}$. The decrease of the saturation magnetization values in comparison to bulk materials is typical for magnetic NPs [60] and it depends on many factors, such as crystal structure, size, shape, and surface properties [61]. The differences in saturation magnetization can also be due to spin disorder at the surface 
of Fe oxide NPs [62]. Moreover, the decrease of $\mathrm{M}_{\mathrm{S}}$ of core-satellite NPs was also caused by a relative decrease of magnetic material per gram of the nanocomposites due to presence of Au satellites.

Table 1. Magnetization values of Fe oxide NPs and core-satellite Au-Fe NPs at $5 \mathrm{~K}$ and $300 \mathrm{~K} . \mathrm{M}_{\mathrm{S}}-$ magnetization saturation, $\mathrm{H}_{(\mathrm{left})}$-intersections on the field axis at increasing and decreasing fields $\left(\mathrm{H}_{(\text {right })}\right), \mathrm{H}_{\mathrm{C}}$-coercive field, $\mathrm{H}_{\mathrm{EB}}$ - exchange bias field.

\begin{tabular}{cccccccc}
\hline Type of NPs & $\mathbf{T}, \mathbf{K}$ & $\mathbf{H}_{(\mathbf{l e f t})}, \mathbf{O e}$ & $\mathbf{H}_{(\mathbf{r i g h t})}, \mathbf{O e}$ & $\mathbf{H}_{\mathbf{C}}, \mathbf{O e}$ & $\mathbf{H}_{\mathrm{EB}}, \mathbf{O e}$ & $\mathbf{M}_{\mathbf{S}}, \mathbf{e m u} \mathbf{g}^{-\mathbf{1}}$ \\
\hline \multirow{2}{*}{ Fe oxide } & 5 & -1572 & 624 & 1098 & -474 & 50.5 \\
\cline { 2 - 8 } & 300 & -109 & 110 & 109.5 & 588.5 & -54.5 & 44.7 \\
\hline \multirow{2}{*}{ Core-satellite } & 5 & -643 & 534 & 133 & 0 & 12.9 \\
\cline { 2 - 7 } & 300 & -134 & 132 & & & 12.3 \\
\hline
\end{tabular}
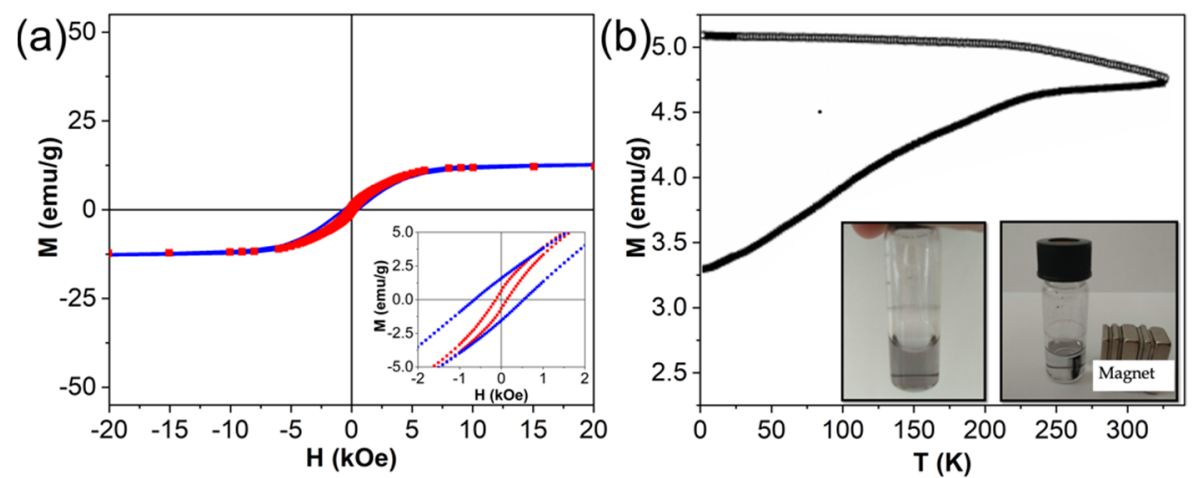

Figure 3. Magnetic characterization of core-satellite Au-Fe NPs. (a) Magnetic hysteresis loops measured at $5 \mathrm{~K}$ (blue) and $300 \mathrm{~K}$ (red). The inset is an expanded view of the low-field region; (b) ZFC-FC curves. The inset represents colloidal solutions of the NPs without magnetic field (left) and attracted by the magnet (right).

It is worth mentioning that magnetization hysteresis loops recorded at $5 \mathrm{~K}$ have a clear negative shift from the origin along the field axis. This observation indicates the presence of an exchange bias field $\left(\mathrm{H}_{\mathrm{EB}}\right)$, which originates from the interface exchange coupling between ferro (ferri)/antiferromagnetic [63]. The presence of exchange bias field can also explain the high value of coercivity for the investigated NPs. Different phases of Fe oxide in the nanocomposites, such as magnetite or maghemite, which are ferrimagnets, and wustite $(\mathrm{FeO})$, which is antiferromagnet, could be responsible for the exchange bias fields [64]. ZFC/FC curves of the core-satellite nanostructures are presented in Figure 3b. No well-defined maximum in the ZFC curve was found. This fact indicates that the NPs had blocking temperatures above $325 \mathrm{~K}$ and exhibit ferromagnetic behavior at room temperature.

Structural and colloidal stability of the synthesized nanocomposites are very important for the projected biomedical applications. To check the stability and find out whether bonds between Fe oxide cores and Au satellites can withstand high ionic strength conditions of physiologically relevant liquids, we incubated the laser-synthesized nanocomposites in aqueous solutions of $1 \mathrm{M} \mathrm{NaCl}$ and in phosphate buffered saline (PBS) at $\mathrm{pH}$ 7.4. Colloidal stability was poor in both liquids: hydrodynamic size of the nanocomposites incubated at $37^{\circ} \mathrm{C}$ doubled after just $5 \mathrm{~min}$ (detailed kinetic of aggregation over the course of $30 \mathrm{~min}$ is shown in Table S1). This result is not surprising since the colloidal stabilization mechanism of bare laser-synthesized NPs is based on electrostatic repulsion, while surface charges are effectively screened in high ionic strength liquids. However, it is very important that the aggregation of the nanostructures was reversible since it was possible to restore optical extinction spectra of the nanocomposites by simple ultrasonication step. The 
reproducibility of the optical extinction spectra after re-dispersion by ultrasonication means that the nanostructures preserved their structural stability in high ionic strength solvents, although they were colloidally unstable.

\subsection{Polymer Coating}

To improve colloidal stability in high ionic strength liquids and to additionally ensure structural stability of the nanocomposites we covered them using several different biocompatible polymer coatings, namely: PEG-silane, PAAC, CMD, PEI, and PAA.

Table S2 summarizes NPs properties after the coating procedure. Efficiency of coating was confirmed by the increase of colloidal stability in PBS buffer and change of $\zeta$-potential of NPs. The best colloidal stability was achieved using negatively charged polymer CMD, while other coatings provided less colloidal stability, or the nanocomposites even aggregated during the coverage procedure (Table S2). The CMD-coated nanocomposites only slightly change their hydrodynamic size after incubation for 24 hours in PBS from $105 \pm 39 \mathrm{~nm}$ to $116 \pm 52 \mathrm{~nm}$ (Figure $4 \mathrm{a}$, Table S2). The coverage by negatively charged CMD polymer changed $\zeta$-potential of the NPs from $+28 \pm 5 \mathrm{mV}$ to $-25 \pm 4.8 \mathrm{mV}$ (Figure $4 \mathrm{~b}$ ) and slightly increased their hydrodynamic size (Figure $4 \mathrm{a}$ ), which additionally confirms the efficiency of the coating procedure.
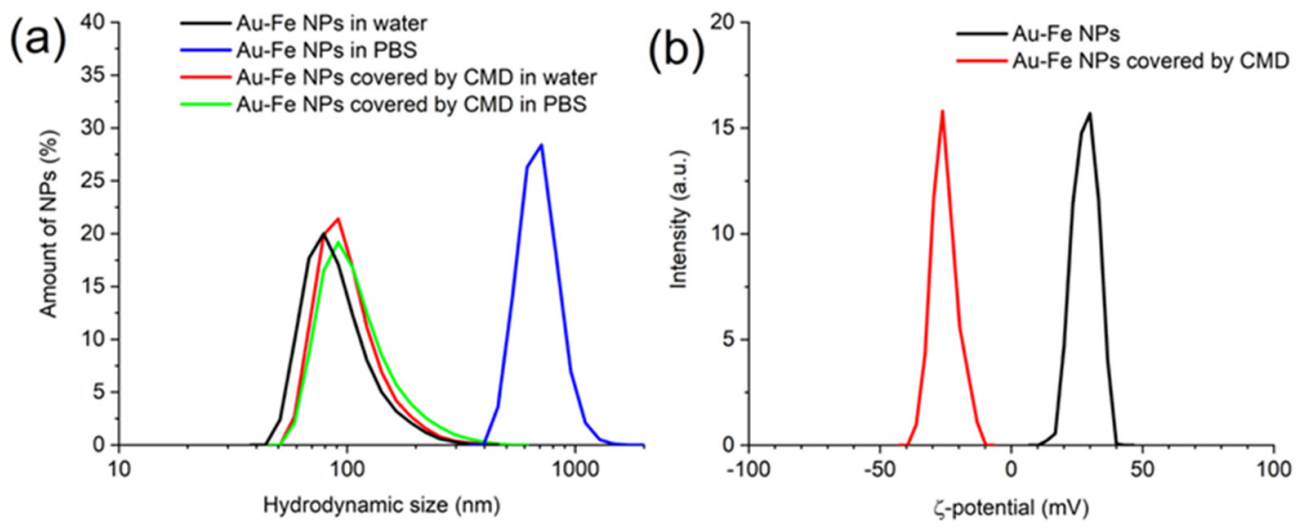

Figure 4. (a) DLS size histograms of the core-satellite Au-Fe NPs after incubation for $24 \mathrm{~h}$ in water and PBS. Black curve-bare NPs incubated in water, blue curve-bare NPs incubated in PBS, red curve-CMD covered NPs incubated in water, green curve-CMD covered NPs incubated in PBS.

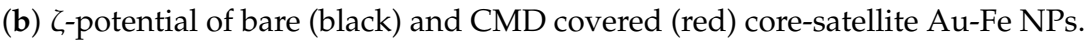

\subsection{Formation Mechanism}

Our experiments showed that $\zeta$-potential was negative for bare laser-synthesized $\mathrm{Au}$ NPs $(-27 \mathrm{mV})$ and positive for both bare Fe oxide NPs $(+30 \mathrm{mV})$ and core-satellite Au-Fe NPs $(+28 \mathrm{mV})$. Therefore, one can suppose that electrostatic interaction can potentially be a major formation mechanism of the nanocomposites. To test this possibility, we separately synthesized bare Au and Fe oxide NPs by a similar laser-ablative procedure (in this case the Fe target was ablated in deionized water in the absence of Au colloids). Then the $\mathrm{Au}$ and Fe-based NPs were mixed in a vial followed by vortexing for several minutes and magnetic separation as described in Materials and Methods section. Finally, we compared the mixture of $\mathrm{Au}$ and Fe oxide NPs with nanocomposites obtained by PLAL. We observed the presence of core-satellite Fe-Au nanostructures in the mixture (Figure S3). However, there was a substantial difference in optical spectra of the samples prepared by PLAL and by NPs mixing as it is shown in Figure S4. The intensity of plasmonic feature of core-satellites NPs normalized to Fe content was five-folds higher for laser-ablated NPs compared to that of NPs prepared by mixing. This highly intense plasmonic signal from laser-synthesized Fe-Au NPs originated from Au NPs attached to Fe oxide cores, because all free Au NPs were removed from colloids by magnetic separation step, while possible differences in concentration of Fe content were accounted by spectra normalization. 
Therefore, the efficiency of the nanocomposite formation was significantly higher for the laser-ablative synthesis than for mixing of oppositely charged Au and Fe oxide NPs. This fact suggests that there are other mechanisms leading to the formation of nanocomposites during ablation of Fe target in Au NPs colloids rather than simple electrostatic interaction of oppositely charged NPs.

In our opinion, the formation of the core-satellites starts from an attraction of small $\mathrm{Au}$ NPs to the forming Fe oxide NPs. The attraction between NPs is originated from several mechanisms including an electrostatic interaction between oppositely charged $\mathrm{Au}$ and $\mathrm{Fe}$ oxide NPs, Van der Waals forces, and hydrophobicity of both Au and Fe oxide NPs. For the formation of stable core-satellites, Au NPs should arrive at the surface of Fe oxide NPs, while the latter are relatively hot. As a result, these small Au NPs fill the potential well at the interface with water and settle down at the surface of Fe oxide NPs. In this way, core-satellites are formed during a limited cooling time of Fe oxide NPs. This explanation is supported by several observations. First, a simple mixing of Au and Fe oxide NPs is less effective for the formation of core-satellite structures than the laser-ablative synthesis. Second, the satellites in the nanocomposites are predominantly formed by the smallest $\mathrm{Au}$ NPs presented in initial gold colloid. Third, larger Fe oxide cores tend to form more core-satellite structures than smaller cores (Figure 2a,b). The second observation can be rationalized by higher speed of NPs Brownian motion of smaller NPs (the speed depends on the size as $r^{-3}$ ). Therefore, small Au NPs move much faster than the large ones and have higher probability of reaching the surface of the Fe oxide NPs during the cooling time. The last observation can be explained by longer cooling time of larger Fe oxide NPs, which gives Au satellites more time to reach the Fe oxide cores. Moreover, note that in our laser ablative setup some of already formed nanostructures are re-irradiated by the laser beam. This re-irradiation can also contribute to the formation of core-satellites in a sintering-like process [65].

Thus, we managed to synthesize stable Au-Fe core-satellite nanocomposites, which combine prominent magnetic response with the generation of plasmonic absorption peak within optical transparency window. At the same time, unique laser-ablative synthesis guarantees purity of the nanocomposites and free access to both $\mathrm{Au}$ and Fe surfaces for further biofunctionalization. There is yet another advantage of the $\mathrm{Fe}_{x} \mathrm{O}_{y} @ \mathrm{Au}$ core-satellite nanocomposites, which potentially can solve the problem of residual accumulation of the nanomaterials in an organism. Fe oxide cores can slowly degrade in physiological conditions and be excreted from an organism, while Au satellites are small enough to be eliminated through renal glomerular filtration mechanism [66]. Therefore, both components of the core-satellites can be naturally removed from an organism, which promises a great advancement in the field of nanomedicine, although this clearance pathway is still to be verified in further research. Combination of these properties makes laser-synthesized $\mathrm{Fe}_{x} \mathrm{O}_{y} @ \mathrm{Au}$ nanocomposites highly suitable for both established and novel theranostic magneto-plasmonic modalities for cancer therapy.

\section{Conclusions}

In conclusion, we synthesized $\mathrm{Fe}_{x} \mathrm{O}_{y} @ \mathrm{Au}$ core-satellite nanocomposites by using a purely physical method of laser ablation in liquids, which insures a high purity of the obtained nanomaterials and the absence of any residual contamination. We experimentally determined optical, structural and magnetic properties of the nanocomposites and elaborated a numerical model, which describes their optical properties. We showed that such nanoformulations combine a strong magnetic response and a good optical extinction within optical transparency window. Additionally, we demonstrated that the surface coating of lasersynthesized nanocomposites with biopolymer CMD can significantly improve its colloidal stability in biologically relevant fluids that is very important for the projected biomedical applications, which are now in progress. From a broad perspective, our research provides a novel extremely pure magneto-plasmonic platform for biomedical nanotheranostics. 
Supplementary Materials: The following are available online at https: / / www.mdpi.com/article/ 10.3390/nano12040649/s1. Figure S1: Typical TEM image of laser-synthesized Au NPs. Figure S2: Calculated extinction spectra of bare Au NPs (red line), Fe oxide NPs (black line), and coresatellite Au-Fe NPs (green line). The following parameters were used for calculations: Au NPs diameter $8 \mathrm{~nm}$; Fe oxide NPs diameter $60 \mathrm{~nm}$, optical constants: $n=2, \mathrm{k}=0.129$; Core-satellite NPs core diameter $60 \mathrm{~nm}$, effective shell thickness $10 \mathrm{~nm}$, optical constants $n=2.4, \mathrm{k}=2.9$. Figure S3: STEM image of nanostructures, obtained by mixing of negatively charged laser-ablated Au NPs and positively charged laser-ablated Fe oxide NPs. Figure S4: Optical extinction spectra of core-satellite nanostructures obtained by mixing of Au NPs and Fe oxide NP (black) and by laser ablation of iron target in colloidal solution of Au NPs (red). Extinction spectrum of laser-ablated Fe oxide NPs was subtracted from both spectra. Table S1: DLS size of the bare core-satellite Au-Fe nanocomposites, incubated in PBS ( $\mathrm{pH} 7.4$ ) at $37^{\circ} \mathrm{C}$. Table S2: DLS size (number distribution) and $\zeta$-potential of the core-satellite Au-Fe NPs, coated with different polymers after incubation for $24 \mathrm{~h}$ in water and PBS.

Author Contributions: A.V.K. and T.E.I. conceived the research. A.A.P., G.T., S.M.K., A.V.K. designed laser fabrication experiments. A.A.P. and G.T. performed the fabrication of core-satellite structures. Z.S.-W. and M.M. performed magnetic measurements. I.V.Z., S.M.D. and A.A.-K. performed physico-chemical characterization of nanomaterials. I.V.Z., S.M.D. developed protocols for coating of nanomaterials by polymers. T.E.I. provided theoretical support to assess properties of core-satellites. A.A.P., Z.S.-W., M.M., G.T., I.V.Z., A.A.-K., S.M.D., S.M.K., T.E.I., A.V.K. analyzed and discussed obtained data. A.A.P. and A.V.K. prepared the manuscript using data from co-authors. A.V.K. guided the project. All authors have read and agreed to the published version of the manuscript.

Funding: The authors gratefully acknowledge the contribution from the Russian Science Foundation (Project 19-72-30012) for the fabrication and functionalization of high-quality samples and a France-Poland trip support provided by the POLONIUM program operated by Campus France Project 37829ZL).

Data Availability Statement: Data is available on reasonable request from the corresponding author.

Conflicts of Interest: The authors declare no conflict of interest.

\section{References}

1. Chen, G.; Roy, I.; Yang, C.; Prasad, P.N. Nanochemistry and Nanomedicine for Nanoparticle-based Diagnostics and Therapy. Chem. Rev. 2016, 116, 2826-2885. [CrossRef] [PubMed]

2. Anselmo, A.C.; Mitragotri, S. Nanoparticles in the clinic. Bioeng. Transl. Med. 2016, 1, 10-29. [CrossRef] [PubMed]

3. Kabashin, A.V.; Singh, A.; Swihart, M.T.; Zavestovskaya, I.N.; Prasad, P.N. Laser-Processed Nanosilicon: A Multifunctional Nanomaterial for Energy and Healthcare. ACS Nano 2019, 13, 9841-9867. [CrossRef] [PubMed]

4. Jain, P.K.; Lee, K.S.; El-Sayed, I.H.; El-Sayed, M.A. Calculated absorption and scattering properties of gold nanoparticles of different size, shape, and composition: Applications in biological imaging and biomedicine. J. Phys. Chem. B 2006, 110, 7238-7248. [CrossRef]

5. Wang, Y.; Yan, B.; Chen, L. SERS Tags: Novel Optical Nanoprobes for Bioanalysis. Chem. Rev. 2013, 113, 1391-1428. [CrossRef]

6. Huang, X.; Jain, P.K.; El-Sayed, I.H.; El-Sayed, M.A. Plasmonic photothermal therapy (PPTT) using gold nanoparticles. Lasers Med. Sci. 2008, 23, 217-228. [CrossRef]

7. Dreaden, E.C.; Alkilany, A.M.; Huang, X.; Murphy, C.J.; El-Sayed, M.A. The golden age: Gold nanoparticles for biomedicine. Chem. Soc. Rev. 2012, 41, 2740-2779. [CrossRef]

8. Bailly, A.; Correard, F.; Popov, A.; Tselikov, G.; Chaspoul, F.; Appay, R.; Al-Kattan, A.; Kabashin, A.V.; Braguer, D.; Esteve, M.-A. In vivo evaluation of safety, biodistribution and pharmacokinetics of laser-synthesized gold nanoparticles. Sci. Rep. 2019, 9, 12890. [CrossRef]

9. Sperling, R.A.; Parak, W.J. Surface modification, functionalization and bioconjugation of colloidal inorganic nanoparticles. Philos. Trans. R Soc. A Math. Phys. Eng. Sci. 2010, 368, 1333-1383. [CrossRef]

10. Rastinehad, A.R.; Anastos, H.; Wajswol, E.; Winoker, J.S.; Sfakianos, J.P.; Doppalapudi, S.K.; Carrick, M.R.; Knauer, C.J.; Taouli, B.; Lewis, S.C.; et al. Gold nanoshell-localized photothermal ablation of prostate tumors in a clinical pilot device study. Proc. Natl. Acad. Sci. USA 2019, 116, 18590-18596. [CrossRef]

11. Gobin, A.M.; Lee, M.H.; Halas, N.J.; James, W.D.; Drezek, R.A.; West, J.L. Near-Infrared Resonant Nanoshells for Combined Optical Imaging and Photothermal Cancer Therapy. Nano Lett. 2007, 7, 1929-1934. [CrossRef] [PubMed]

12. Nguyen, V.P.; Qian, W.; Li, Y.; Liu, B.; Aaberg, M.; Henry, J.; Zhang, W.; Wang, X.; Paulus, Y.M. Chain-like gold nanoparticle clusters for multimodal photoacoustic microscopy and optical coherence tomography enhanced molecular imaging. Nat. Commun. 2021, 12, 34. [CrossRef] [PubMed] 
13. Sokolov, K.; Follen, M.; Aaron, J.; Pavlova, I.; Malpica, A.; Lotan, R.; Richards-Kortum, R. Real-time vital optical imaging of precancer using anti-epidermal growth factor receptor antibodies conjugated to gold nanoparticles. Cancer Res. 2003, 63, 1999-2004. [PubMed]

14. Lu, A.-H.; Salabas, E.L.; Schüth, F. Magnetic Nanoparticles: Synthesis, Protection, Functionalization, and Application. Angez. Chemie Int. Ed. 2007, 46, 1222-1244. [CrossRef] [PubMed]

15. Sun, C.; Lee, J.S.H.; Zhang, M. Magnetic nanoparticles in MR imaging and drug delivery. Adv. Drug Deliv. Rev. 2008, 60, 1252-1265. [CrossRef]

16. Borlido, L.; Azevedo, A.M.; Roque, A.C.A.; Aires-Barros, M.R. Magnetic separations in biotechnology. Biotechnol. Adv. 2013, 31, 1374-1385. [CrossRef]

17. Ulbrich, K.; Holá, K.; Šubr, V.; Bakandritsos, A.; Tuček, J.; Zbořil, R. Targeted Drug Delivery with Polymers and Magnetic Nanoparticles: Covalent and Noncovalent Approaches, Release Control, and Clinical Studies. Chem. Rev. 2016, 116, 5338-5431. [CrossRef]

18. Hergt, R.; Dutz, S.; Müller, R.; Zeisberger, M. Magnetic particle hyperthermia: Nanoparticle magnetism and materials development for cancer therapy. J. Phys. Condens. Matter 2006, 18, S2919-S2934. [CrossRef]

19. Tomitaka, A.; Arami, H.; Raymond, A.; Yndart, A.; Kaushik, A.; Jayant, R.D.; Takemura, Y.; Cai, Y.; Toborek, M.; Nair, M. Development of magneto-plasmonic nanoparticles for multimodal image-guided therapy to the brain. Nanoscale 2017, 9, 764-773. [CrossRef]

20. Bandyopadhyay, S.; Singh, G.; Sandvig, I.; Sandvig, A.; Mathieu, R.; Anil Kumar, P.; Glomm, W.R. Synthesis and in vitro cellular interactions of superparamagnetic iron nanoparticles with a crystalline gold shell. Appl. Surf. Sci. 2014, 316, 171-178. [CrossRef]

21. Caruntu, D.; Cushing, B.L.; Caruntu, G.; O'Connor, C.J. Attachment of Gold Nanograins onto Colloidal Magnetite Nanocrystals. Chem. Mater. 2005, 17, 3398-3402. [CrossRef]

22. Ye, M.; Wei, Z.; Hu, F.; Wang, J.; Ge, G.; Hu, Z.; Shao, M.; Lee, S.-T.; Liu, J. Fast assembling microarrays of superparamagnetic $\mathrm{Fe}_{3} \mathrm{O}_{4} @$ Au nanoparticle clusters as reproducible substrates for surface-enhanced Raman scattering. Nanoscale 2015, 7, 13427-13437. [CrossRef] [PubMed]

23. León Félix, L.; Sanz, B.; Sebastián, V.; Torres, T.E.; Sousa, M.H.; Coaquira, J.A.H.; Ibarra, M.R.; Goya, G.F. Gold-decorated magnetic nanoparticles design for hyperthermia applications and as a potential platform for their surface-functionalization. Sci. Rep. 2019, 9, 4185. [CrossRef] [PubMed]

24. Levin, C.S.; Hofmann, C.; Ali, T.A.; Kelly, A.T.; Morosan, E.; Nordlander, P.; Whitmire, K.H.; Halas, N.J. Magnetic-plasmonic core-shell nanoparticles. ACS Nano 2009, 3, 1379-1388. [CrossRef]

25. Wang, C.; Xu, S.; Zhang, K.; Li, M.; Li, Q.; Xiao, R.; Wang, S. Streptomycin-modified $\mathrm{Fe}_{3} \mathrm{O}_{4}-\mathrm{Au} @ \mathrm{Ag}$ core-satellite magnetic nanoparticles as an effective antibacterial agent. J. Mater. Sci. 2017, 52, 1357-1368. [CrossRef]

26. Araújo, J.E.; Lodeiro, C.; Capelo, J.L.; Rodríguez-González, B.; dos Santos, A.A.; Santos, H.M.; Fernández-Lodeiro, J. Novel nanocomposites based on a strawberry-like gold- coated magnetite (Fe@Au) for protein separation in multiple myeloma serum samples. Nano Res. 2015, 8, 1189-1198. [CrossRef]

27. Seino, S.; Kinoshita, T.; Otome, Y.; Okitsu, K.; Nakagawa, T.; Yamamoto, T.A. Magnetic Composite Nanoparticle of Au/ $\gamma$-Fe ${ }_{2} \mathrm{O}_{3}$ Synthesized by Gamma-Ray Irradiation. Chem. Lett. 2003, 32, 690-691. [CrossRef]

28. Itina, T.E.; Gouriet, K.; Zhigilei, L.V.; Noël, S.; Hermann, J.; Sentis, M. Mechanisms of small clusters production by short and ultra-short laser ablation. Appl. Surf. Sci. 2007, 253, 7656-7661. [CrossRef]

29. Kabashin, A.V.; Meunier, M. Laser-induced treatment of silicon in air and formation of $\mathrm{Si} / \mathrm{SiO}_{x}$ photoluminescent nanostructured layers. Mater. Sci. Eng. B 2003, 101, 60-64. [CrossRef]

30. Fojtik, A.; Henglein, A. Laser Ablation of Films and Suspended Particles in a Solvent: Formation of Cluster and Colloid Solutions. Ber. Bunsenges. Phys. Chem. 1993, 97, 252. [CrossRef]

31. Zhang, D.; Gökce, B.; Barcikowski, S. Laser Synthesis and Processing of Colloids: Fundamentals and Applications. Chem. Rev. 2017, 117, 3990-4103. [CrossRef] [PubMed]

32. Kabashin, A.V.; Timoshenko, V.Y. What theranostic applications could ultrapure laser-synthesized Si nanoparticles have in cancer? Nanomedicine 2016, 11, 2247-2250. [CrossRef] [PubMed]

33. Kabashin, V.K.; Meunier, M. Synthesis of Colloidal Nanoparticles during Femtosecond Laser Ablation of Gold in Water. J. Appl. Phys. 2003, 94, 7941. [CrossRef]

34. Kabashin, A.V.; Meunier, M.; Kingston, C.; Luong, J.H.T. Fabrication and Characterization of Gold Nanoparticles by Femtosecond Laser Ablation in an Aqueous Solution of Cyclodextrins. J. Phys. Chem. B 2003, 107, 4527-4531. [CrossRef]

35. Sajti, C.L.; Giorgio, S.; Khodorkovsky, V.; Marine, W. Femtosecond laser synthesized nanohybrid materials for bioapplications. Appl. Surf. Sci. 2007, 253, 8111-8114. [CrossRef]

36. Baati, T.; Al-Kattan, A.; Esteve, M.-A.; Njim, L.; Ryabchikov, Y.; Chaspoul, F.; Hammami, M.; Sentis, M.; Kabashin, A.V.; Braguer, D. Ultrapure laser-synthesized Si-based nanomaterials for biomedical applications: In vivo assessment of safety and biodistribution. Sci. Rep. 2016, 6, 25400. [CrossRef]

37. Popov, A.A.; Tselikov, G.; Dumas, N.; Berard, C.; Metwally, K.; Jones, N.; Al-Kattan, A.; Larrat, B.; Braguer, D.; Mensah, S.; et al. Laser- synthesized TiN nanoparticles as promising plasmonic alternative for biomedical applications. Sci. Rep. 2019, 9, 1194. [CrossRef] 
38. Zelepukin, I.V.; Popov, A.A.; Shipunova, V.O.; Tikhonowski, G.V.; Mirkasymov, A.B.; Popova-Kuznetsova, E.A.; Klimentov, S.M.; Kabashin, A.V.; Deyev, S.M. Laser-synthesized TiN nanoparticles for biomedical applications: Evaluation of safety, biodistribution and pharmacokinetics. Mater. Sci. Eng. C 2021, 120, 111717. [CrossRef]

39. Lim, C.-K.; Popov, A.A.; Tselikov, G.; Heo, J.; Pliss, A.; Kim, S.; Kabashin, A.V.; Prasad, P.N. Organic Solvent and Surfactant Free Fluorescent Organic Nanoparticles by Laser Ablation of Aggregation-Induced Enhanced Emission Dyes. Adv. Opt. Mater. 2018 6, 1800164. [CrossRef]

40. Wagener, P.; Jakobi, J.; Rehbock, C.; Chakravadhanula, V.S.K.; Thede, C.; Wiedwald, U.; Bartsch, M.; Kienle, L.; Barcikowski, S. Solvent-surface interactions control the phase structure in laser-generated iron-gold core-shell nanoparticles. Sci. Rep. 2016, 6, 23352. [CrossRef]

41. Tymoczko, A.; Kamp, M.; Prymak, O.; Rehbock, C.; Jakobi, J.; Schürmann, U.; Kienle, L.; Barcikowski, S. How the crystal structure and phase segregation of Au-Fe alloy nanoparticles are ruled by the molar fraction and size. Nanoscale 2018, 10, 16434-16437. [CrossRef] [PubMed]

42. Amendola, V.; Scaramuzza, S.; Litti, L.; Meneghetti, M.; Zuccolotto, G.; Rosato, A.; Nicolato, E.; Marzola, P.; Fracasso, G.; Anselmi, C.; et al. Magneto-plasmonic Au-Fe alloy nanoparticles designed for multimodal SERS-MRI-CT imaging. Small 2014, 10, 2476-2486. [CrossRef] [PubMed]

43. Amendola, V.; Scaramuzza, S.; Agnoli, S.; Polizzi, S.; Meneghetti, M. Strong dependence of surface plasmon resonance and surface enhanced Raman scattering on the composition of Au-Fe nanoalloys. Nanoscale 2014, 6, 1423-1433. [CrossRef] [PubMed]

44. Kögler, M.; Ryabchikov, Y.V.; Uusitalo, S.; Popov, A.; Popov, A.; Tselikov, G.; Välimaa, A.-L.; Al-Kattan, A.; Hiltunen, J.; Laitinen, R.; et al. Bare laser-synthesized Au-based nanoparticles as nondisturbing surface-enhanced Raman scattering probes for bacteria identification. J. Biophotonics 2018, 11, e201700225. [CrossRef] [PubMed]

45. Ryabchikov, Y.; Popov, A.A.; Sentis, M.; Timoshenko, V.Y.; Kabashin, A.V. Structural properties of gold-silicon nanohybrids formed by femtosecond laser ablation in water at different fluences. In Synthesis and Photonics of Nanoscale Materials XIII; Kabashin, A.V., Geohegan, D.B., Dubowski, J.J., Eds.; SPIE: San-Francisco, CA, USA, 2016; Volume 9737, p. 97370F.

46. Sylvestre, J.P.; Poulin, S.; Kabashin, A.V.; Sacher, E.; Meunier, M.; Luong, J.H.T. Surface chemistry of gold nanoparticles produced by laser ablation in aqueous media. J. Phys. Chem. B 2004, 108, 16864-16869. [CrossRef]

47. Rehbock, C.; Merk, V.; Gamrad, L.; Streubel, R.; Barcikowski, S. Size control of laser-fabricated surfactant-free gold nanoparticles with highly diluted electrolytes and their subsequent bioconjugation. Phys. Chem. Chem. Phys. 2013, 15, 3057-3067. [CrossRef]

48. Mie, G. Beiträge zur Optik trüber Medien, speziell kolloidaler Metallösungen. Ann. Phys. 1908, 330, 377-445. [CrossRef]

49. Wriedt, T. Light scattering theories and computer codes. J. Quant. Spectrosc. Radiat. Transf. 2009, 110, 833-843. [CrossRef]

50. Peña, O.; Pal, U. Scattering of electromagnetic radiation by a multilayered sphere. Comput. Phys. Commun. 2009, 180, 2348-2354. [CrossRef]

51. Peña-Rodríguez, O.; González Pérez, P.P.; Pal, U. MieLab: A Software Tool to Perform Calculations on the Scattering of Electromagnetic Waves by Multilayered Spheres. Int. J. Spectrosc. 2011, 2011, 1-10. [CrossRef]

52. Tymoczko, A.; Kamp, M.; Rehbock, C.; Kienle, L.; Cattaruzza, E.; Barcikowski, S.; Amendola, V. One-step synthesis of Fe-Au core-shell magnetic-plasmonic nanoparticles driven by interface energy minimization. Nanoscale Horiz. 2019, 4, $1326-1332$. [CrossRef]

53. Prodan, E.; Radloff, C.; Halas, N.J.; Nordlander, P. A Hybridization Model for the Plasmon Response of Complex Nanostructures. Science 2003, 302, 419-422. [CrossRef]

54. Mafuné, F.; Kohno, J.; Takeda, Y.; Kondow, T. Formation of Gold Nanonetworks and Small Gold Nanoparticles by Irradiation of Intense Pulsed Laser onto Gold Nanoparticles. J. Phys. Chem. B 2003, 107, 12589-12596. [CrossRef]

55. Jain, P.K.; Huang, W.; El-Sayed, M.A. On the Universal Scaling Behavior of the Distance Decay of Plasmon Coupling in Metal Nanoparticle Pairs: A Plasmon Ruler Equation. Nano Lett. 2007, 7, 2080-2088. [CrossRef]

56. Weiglhofer, W.S.; Lakhtakia, A.; Michel, B. Maxwell Garnett and Bruggeman formalisms for a particulate composite with bianisotropic host medium. Microw. Opt. Technol. Lett. 1997, 15, 263-266. [CrossRef]

57. Levy, O.; Stroud, D. Maxwell Garnett theory for mixtures of anisotropic inclusions: Application to conducting polymers. Phys. Rev. B 1997, 56, 8035-8046. [CrossRef]

58. Lu, H.M.; Zheng, W.T.; Jiang, Q. Saturation magnetization of ferromagnetic and ferrimagnetic nanocrystals at room temperature. J. Phys. D Appl. Phys. 2007, 40, 320-325. [CrossRef]

59. Cao, D.; Li, H.; Pan, L.; Li, J.; Wang, X.; Jing, P.; Cheng, X.; Wang, W.; Wang, J.; Liu, Q. High saturation magnetization of $\gamma-\mathrm{Fe}_{2} \mathrm{O}_{3}$ nano-particles by a facile one-step synthesis approach. Sci. Rep. 2016, 6, 32360. [CrossRef]

60. Saxena, N.; Singh, M. Efficient synthesis of superparamagnetic magnetite nanoparticles under air for biomedical applications. $J$. Magn. Magn. Mater. 2017, 429, 166-176. [CrossRef]

61. Wu, W.; Wu, Z.; Yu, T.; Jiang, C.; Kim, W.-S. Recent progress on magnetic iron oxide nanoparticles: Synthesis, surface functional strategies and biomedical applications. Sci. Technol. Adv. Mater. 2015, 16, 023501. [CrossRef]

62. Shen, L.; Qiao, Y.; Guo, Y.; Meng, S.; Yang, G.; Wu, M.; Zhao, J. Facile co-precipitation synthesis of shape-controlled magnetite nanoparticles. Ceram. Int. 2014, 40, 1519-1524. [CrossRef]

63. Swiatkowska-Warkocka, Z.; Kawaguchi, K.; Wang, H.; Katou, Y.; Koshizaki, N. Controlling exchange bias in $\mathrm{Fe}_{3} \mathrm{O}_{4} / \mathrm{FeO}$ composite particles prepared by pulsed laser irradiation. Nanoscale Res. Lett. 2011, 6, 226. [CrossRef] 
64. Chalasani, R.; Vasudevan, S. Form, Content, and Magnetism in Iron Oxide Nanocrystals. J. Phys. Chem. C 2011, 115, 18088-18093. [CrossRef]

65. Goudeli, E.; Pratsinis, S.E. Crystallinity dynamics of gold nanoparticles during sintering or coalescence. AIChE J. 2016, 62, 589-598. [CrossRef]

66. Soo Choi, H.; Liu, W.; Misra, P.; Tanaka, E.; Zimmer, J.P.; Itty Ipe, B.; Bawendi, M.G.; Frangioni, J.V. Renal clearance of quantum dots. Nat. Biotechnol. 2007, 25, 1165-1170. [CrossRef] 